\title{
Doença de depósito lisossomal induzida pelo consumo de Ipomoea verbascoidea (Convolvulaceae) em caprinos no semiárido de Pernambuco ${ }^{1}$
}

\author{
Dayane D.C.C. Lima ${ }^{2}$, Raquel F. Albuquerque², Brena P. Rocha ${ }^{3}$, Maria Edna G. Barros ${ }^{4}$, \\ Dale R. Gardner ${ }^{5}$, Rosane M.T. Medeiros ${ }^{6}$, Franklin Riet-Correa ${ }^{6}$ e Fábio S. Mendonça ${ }^{7 *}$
}

\begin{abstract}
Lima D.D.C.C., Albuquerque R.F., Rocha B.P., Barros M.E.G., Gardner D.R., Medeiros R.M.T., Riet-Correa F. \& Mendonça F.S. 2013. [Lysosomal storage disease induced by the consumption of Ipomoea verbascoidea (Convolvulaceae) in goats at northeastern Brazil.] Doença de depósito lisossomal induzida pelo consumo de Ipomoea verbascoidea (Convolvulaceae) em caprinos no semiárido de Pernambuco. Pesquisa Veterinária Brasileira 33(7):867-872. Departamento de Morfologia e Fisiologia Animal, Universidade Federal Rural de Pernambuco, Rua Dom Manuel Medeiros s/n, Dois Irmãos, Recife, PE 52171-900, Brazil. E-mail: mendonca@dmfa.ufrpe.br

The aim of this paper was to reproduce the poisoning of Ipomoea verbascoidea in goats and describe the epidemiological, clinical and pathological aspects of spontaneous poisoning by this plant in Pernambuco. For this, we studied the epidemiology of the disease in seven municipalities in the semiarid region of the State. Three spontaneously poisoned goats were examined and then euthanized and necropsied (Group I). To reproduce the disease, the dried leaves of $I$. verbascoidea containing $0.02 \%$ swainsonine were supplied at doses of $4 \mathrm{~g} / \mathrm{kg}(0.8 \mathrm{mg}$ swainsonine $/ \mathrm{kg}$ ) to two groups of three animals. The goats in Group II received daily doses of the plant during 40 days and were euthanized on the $41^{\text {st }}$ day of the experiment. Goats from Group III received daily doses of the plant during 55 days and were euthanized on the $120^{\text {th }}$ day of the experiment. Other three goats constituted the control group (Group IV). In experimental groups, the brain lesions were evaluated by histopathology; additionally the cerebellar lesions were evaluated by morphometry, by measuring the molecular layer thickness, the number of Purkinje cells and the area of the cell bodies of these cells. The main clinical signs and microscopic lesions in goats poisoned were similar to those reported by swainsonine containing plants. In goats of GII and GIII, the first nervous signs were observed between $22^{\text {th }}$ and $29^{\text {th }}$ days; clinically, the disease developed by these animals was similar to the spontaneous cases. The goats of GIII did not recover from the neurologic signs. These results show that the consumption of the plant by 26-28 days after observation of the first clinical signs is enough to cause irreversible damage. By morphometric analysis, the molecular layer of the cerebellum of the goats of Group I and III were thinner than those of goats in the control group, and Purkinje neurons were atrophic. It is suggested that these changes are responsible for the neurological picture observed in goats that stop eating the plant and have sequelae of poisoning.
\end{abstract}

INDEX TERMS: Poisonous plants, plant poisoning, lysosomal storage disease, swainsonine, goats, Ipomoea verbascoidea, Convolvulaceae.

\footnotetext{
${ }^{1}$ Recebido em 13 de maio de 2013.

Aceito para publicação em 4 de junho de 2013.

${ }^{2}$ Programa de Pós-Graduação em Ciência Veterinária, Departamento de Medicina Veterinária, Universidade Federal Rural do Pernambuco (UFRPE), Rua Dom Manuel Medeiros s/n, Dois Irmãos, Recife, PE 52171-900, Brasil.

${ }^{3}$ Departamento de Medicina Veterinária, Curso de Medicina Veterinária, UFRPE, Recife, PE.

${ }^{4}$ Programa de Pós-Graduação em Biociência Animal, Departamento de
}

Morfologia e Fisiologia Animal, Universidade Federal Rural do Pernambuco, Recife, PE.

${ }^{5}$ USDA-ARS Poisonous Plants Research Laboratory, Logan, UT 84341, USA.

${ }^{6}$ Hospital Veterinário, Centro de Saúde e Tecnologia Rural (CSTR), Universidade Federal de Campina Grande (UFCG), Patos, PB 58700-000, Brasil.

${ }^{7}$ Laboratório de Diagnóstico Animal, Departamento de Morfologia e Fisiologia Animal (DMFA), UFRPE, Rua Dom Manuel Medeiros s/n, Dois Irmãos, Recife, PE. *Autor para correspondência: mendonca@dmfa.ufrpe.br 
RESUMO.- 0 objetivo deste trabalho foi reproduzir a intoxicação por Ipomoea verbascoidea em caprinos e descrever os aspectos epidemiológicos, clínicos e histopatológicos da intoxicação espontânea por essa planta no Estado de Pernambuco. Para isso, realizou-se o acompanhamento da epidemiologia da doença em sete municípios do semiárido pernambucano. Três caprinos espontaneamente intoxicados foram examinados e, em seguida eutanasiados e necropsiados (Grupo I). Para reproduzir experimentalmente a doença, as folhas secas de I. verbascoidea contendo $0,02 \%$ de swainsonina, foram fornecidas na dose de $4 \mathrm{~g} / \mathrm{kg}(0,8 \mathrm{mg}$ de swainsonina/kg) a dois grupos de três animais. Os caprinos do Grupo II receberam a planta diariamente por 40 dias e foram eutanasiados no $41^{\circ}$ dia de experimento. Os caprinos do Grupo III receberam a planta diariamente por 55 dias e foram eutanasiados no $120^{\circ}$ dia de experimento. Outros três caprinos constituíram o grupo controle (Grupo IV). Nos grupos experimentais, as lesões encefálicas foram avaliadas por histopatologia e adicionalmente avaliaram-se as lesões cerebelares por morfometria, mediante mensuração da espessura da camada molecular, do número de neurônios de Purkinje e da área dos corpos celulares dessas células. Os principais sinais clínicos e lesões microscópicas foram semelhantes aos previamente reportados em animais intoxicados por plantas que contem swainsonina. Nos caprinos do GII e GIII, os primeiros sinais clínicos foram observados entre o $22^{\circ}$ e $29^{\circ}$ dia de experimento; clinicamente a doença desenvolvida por esses animais foi semelhante aos casos espontâneos. Nenhum dos caprinos do GIII se recuperou dos sinais neurológicos. Esse resultado evidencia que o consumo da planta por 26-28 dias após a observação dos primeiros sinais clínicos é suficiente para provocar lesões irreversíveis. Pela análise morfométrica, a camada molecular do cerebelo dos caprinos do Grupo I e III eram mais delgadas que às dos caprinos do grupo controle, e os neurônios de Purkinje estavam atróficos. Sugere-se que essas alterações sejam responsáveis pelo quadro clínico neurológico observado nos caprinos que deixam de ingerir a planta e apresentam seqüelas da intoxicação.

TERMOS DE INDEXAÇÃO: Plantas tóxicas, intoxicação por planta, doenças de depósito lisossomal, swainsonina, caprinos, Ipomoea verbascoidea, Convolvulaceae.

\section{INTRODUÇÃO}

Ipomoea verbascoidea Choisy (Convolvulacea), um arbusto sub-ereto com caules decumbentes, com até $5 \mathrm{~m}$ de altura, com folhas oblongas ou circulares, flores de coloração lilás, solitárias ou agrupadas em número de 3 , com sementes ovóides e de coloração acastanhada, é conhecida popularmente como "jetirana", "orelha-de-onça" ou "batata-de-peba" no nordeste do Brasil. Essa liana contém o alcaloide 1,2,8-triol indolizidina (swainsonina) e foi recentemente descrita como responsável por surtos de doença de depósito lisossomal (DDL) afetando caprinos no Estado de Pernambuco (Mendonça et al. 2012).

As plantas que contêm swainsonina e causam desordens neurológicas associadas com armazenamento de oligossacarídeos compõem um grupo muito importante de plantas tóxicas no Brasil (Oliveira et al. 2013). No semiárido nordestino, as intoxicações por plantas que contém esse alcaloide ocorrem principalmente em caprinos (Balogh et al. 1999, Barbosa et al. 2006, Colodel et al. 2002, Dantas et al. 2007, Mendonça et al. 2011, Mendonça et al. 2012), mas intoxicações em bovinos, ovinos (Guedes et al. 2007) e equinos (Dantas et al. 2007, Assis et al. 2010) podem ocorrer ocasionalmente. Os sinais clínicos consistem principalmente em distúrbios neurológicos, embora alterações endócrinas e reprodutivas também possam ocorrer (Oliveira et al. 2011). As alterações neurológicas observadas são principalmente de origem cerebelar; evidenciadas pela perda do equilíbrio, seguida de quedas quando os animais são estressados, ataxia, hipermetria, dismetria, nistagmo, marcha lateral e tremores de cabeça e pescoço. Após sofrerem quedas, os animais têm dificuldade em se levantar e podem apresentar espasticidade dos membros pélvicos. Outras alterações, tais como sonolência, emagrecimento progressivo, pêlos arrepiados, opacos e quebradiços também podem ser observados (Oliveira et al. 2009, Tokarnia et al. 2012, Oliveira et al. 2013).

Neste trabalho são descritos os aspectos epidemiológicos, sinais clínicos e achados histopatológicos da intoxicação espontânea e experimental por I. verbascoidea em caprinos.

\section{MATERIAL E MÉTODOS}

\section{Epidemiologia}

O estudo epidemiológico foi realizado na microrregião do Sertão do Moxotó, Estado de Pernambuco (Fig.1) nos meses de março a maio dos anos 2010-2012. O clima predominante nos Municípios que compreendem essa microrregião é o semiárido, com temperaturas elevadas e chuvas escassas e mal distribuídas. A vegetação característica é a caatinga (IBGE 2010).

Para determinar a ocorrência das intoxicações por Ipomoea verbascoidea nessa microrregião foram entrevistados 35 indivíduos ligados a atividades agropecuárias, dentre eles produtores, médicos veterinários e zootecnistas, sendo 5 por município. Para as entrevistas foi utilizado um formulário similar ao utilizado por Silva et al. (2006), com espaço para identificação do entrevistado e perguntas sobre a ocorrência de plantas tóxicas e surtos na região estudada.

\section{Intoxicação espontânea}

Três caprinos machos, com idade aproximada de 12 meses, pesando em média $20 \mathrm{~kg}$, naturalmente intoxicados por I. verbascoidea (Grupo I: Caprinos 1-3) foram examinados mais detalhadamente, eutanasiados e necropsiados. Após fixação, o sistema nervoso central (SNC) foi seccionado em cortes transversais de 4-5 $\mathrm{mm}$. Do SNC foram coletadas amostras da medula espinhal cervical, torácica e lombar, medula oblonga, ponte, colículo rostral, tálamo, córtex telencefálico, pedúnculos cerebelares, cerebelo e gânglio trigêmeo. Também se coletou amostras de tireóide, pâncreas, rins e fígado. Esses fragmentos foram processados de acordo com os métodos de rotina, corados pela hematoxina-eosina e avaliados histopatologicamente.

\section{Intoxicação experimental}

As folhas de I. verbascodiea foram coletadas no município de Sertânia-PE, entre os meses de março e maio de 2011. As folhas foram secas à sombra, trituradas e acondicionadas em sacos de 
nylon para posterior fornecimento aos caprinos do experimento. 0 peso das folhas secas representou $40 \%$ das folhas verdes. A concentração de swainsonina e calysteginas nas folhas dessecadas de I. verbascoidea foi mensurada por cromatografia líquida acoplada à espectrometria de massa, de acordo com os procedimentos descritos por Gardner et al. (2001).

No experimento foram utilizados nove caprinos machos; clinicamente sadios, com idade entre 6 e 9 meses, e peso entre 16$21 \mathrm{~kg}$. Trinta dias antes do início dos experimentos os caprinos receberam medicação antiparasitária, foram numerados ao acaso e mantidos em baias individuais, visando à adaptação e observação clínica prévia. No início da manhã, recebiam ração comercial, suplemento mineral, feno de tifton (Cynodon dactylon) e água ad libitum.

Após o período de adaptação os caprinos foram divididos em três grupos: Grupo II, constituído pelos Caprinos 4-6 [caprinos que receberam misturadas à ração $I$. verbascoidea dessecada contendo $0,02 \%$ de swainsonina na dose de $4 \mathrm{~g} / \mathrm{kg}(0,8 \mathrm{mg}$ de swainsonina/kg) durante 40 dias e eutanasiados no $41^{\circ}$ dia de experimento]. Grupo III, constituído pelos Caprinos 7-9 [caprinos que receberam misturadas à ração $I$. verbascoidea dessecada contendo $0,02 \%$ de swainsonina na dose $\mathrm{de} 4 \mathrm{~g} / \mathrm{kg}(0,8 \mathrm{mg}$ de swainsonina/kg) durante 55 dias e eutanasiados no $120^{\circ}$ dia de experimento]. Grupo IV (grupo controle), constituído pelos Caprinos 10-12 (caprinos que ficaram sob as mesmas condições de manejo que os caprinos dos Grupos II e III, porém não receberam I. verbascoidea na dieta).

Os caprinos foram avaliados clinicamente uma vez ao dia segundo descreveram Smith \& Sherman (1994), quanto ao seu estado geral, comportamento, atitude, estado mental, coordenação, postura da cabeça, movimentação, apetite, cor das mucosas, vasos espisclerais, temperatura retal, freqüências cardíaca e respiratória, forma do abdômen, motilidade retículo-ruminal, exame físico das fezes, urina e aparência da pele. Também foram realizados o Head Raising Test (HR test) e o Stand Up Test. O HR test consiste em levantar a cabeça do animal forçando-a para trás no sentido crânio caudal, mantendo essa posição durante um minuto e em seguida soltando-a subitamente para verificar se há perda de equilíbrio (Pienaar et al. 1976). O Stand Up Test consiste em manter o animal em decúbito lateral por um minuto e em seguida soltá-lo para avaliar se existe dificuldade em se levantar (Barbosa et al. 2006).

Ao fim do período experimental os caprinos foram eutanasiados e necropsiados. Os procedimentos de coleta e processamento de fragmentos do SNC e de tecidos das cavidades torácica e abdominal foram realizados conforme descrito anteriormente nos casos das intoxicações espontâneas.

\section{Morfometria cerebelar}

A análise morfométrica do cerebelo foi realizada mediante secção de um fragmento longitudinal do verme cerebelar dos 12 caprinos estudados. Para tanto, foram fotografadas 20 folhas cerebelares em microscópio óptico Nikon Eclipse 50i com câmera Samsung SV3 acoplada, utilizando-se a objetiva de 10x. As imagens geradas foram lançadas no software de processamento e análise de imagem (Image Lab 3.000) para a mensuração da espessura da camada molecular nas regiões do ápice e faces laterais de cada folha. Também foram contados todos os neurônios de Purkinge de cada uma das 20 folhas cerebelares e mensurada a área dos corpos celulares dessas células, utilizando-se para isto, o software Motic Image Plus 2.0. Para análise estatística de todos os dados foi aplicado o teste de normalidade através do software ASSISTAT Versão 7.6 beta (2011). Para os dados referentes à mensuração da espessura da camada molecular foi empregado o Post-hoc de Tukey, com nível de 5\% de significância e para a avaliação do teste comparati- vo do número de neurônios de Purkinje e da área do corpo celular dessas células, foi utilizado o Post-hoc t de Student ( $\mathrm{p}<0.05)$.

\section{RESULTADOS}

\section{Epidemiologia e intoxicação espontânea}

Em Custódia, Ipomoea verbascoidea foi mencionada uma vez como responsável pela morte de três caprinos. Em Arcoverde, Betânia e Ibimirim nenhum surto foi relatado, porém a equipe do Laboratório de Diagnóstico Animal (LDA) da UFRPE encontrou a planta nos três municípios. Em três propriedades rurais de Sertânia, no período chuvoso dos anos de 2010-2011 a planta causou a morte de 25 caprinos. Em todos esses cinco municípios, quantidades mais significativas da planta eram encontradas em regiões de caatinga alta e onde a vegetação estava mais preservada. Nos Municípios de Inajá e Manarí a intoxicação pela planta não foi reconhecida e a planta também não foi encontrada. No ano de 2012 nenhum caprino foi afetado pela doença na microrregião estudada.

Nos Caprinos 1-3, os principais sinais clínicos eram neurológicos. Nos Caprinos 1-2, os sinais nervosos eram moderados e consistiam em ataxia, marcha lateral e reações posturais lentas; apesar disso, os caprinos apresentavam bom escore corporal. No Caprino 3 os sinais nervosos eram mais discretos e consistiam unicamente em lentidão e sonolência. Não foram observadas alterações macroscópicas nos caprinos necropsiados. Microscopicamente só havia lesões no encéfalo do caprino C1 que consistiam em fina vacuolização citoplasmática em neurônios de Purkinje e neurônios do núcleo cerebelar, ponte e medula oblonga. Esferóides axonais foram observados na camada granular do cerebelo. Nesse caprino, vacuolizações citoplasmáticas foram também observadas em neurônios do córtex cerebral, gânglio trigêmeo e medula espinhal. Também havia vacuolização citoplasmática em células epiteliais dos túbulos renais, células acinares pancreáticas, foliculares da tireoide e hepatócitos.

\section{Intoxicação experimental}

Nas duas primeiras semanas em que a planta foi fornecida aos Caprinos 4-9, o HR test e o Stand Up Test resulta-

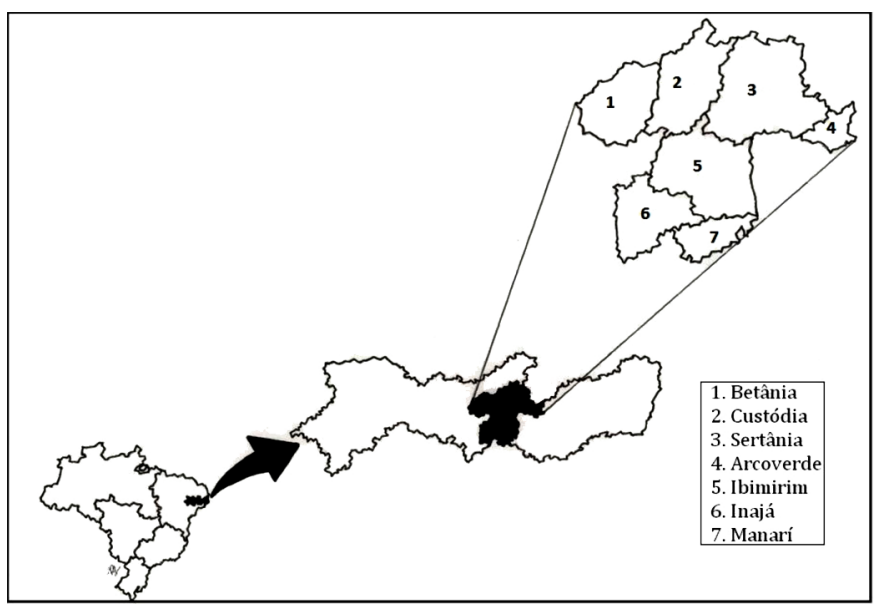

Fig.1. Mapa geográfico do Brasil destacando o Estado de Pernambuco, o Sertão do Moxotó e, em maior aumento, os Municípios estudados. 
ram negativos. Os primeiros sinais clínicos foram observados no $22^{\circ}$ dia de experimento, no Caprino 5. No restante dos caprinos os sinais clínicos foram observados entre $27^{\circ}$ e 29o dia de experimento. Esses sinais consistiram em deficiências proprioceptivas, com alterações de equilíbrio, postura e coordenação. No Caprino 5, os sinais foram mais intensos e de evolução mais rápida. Nos outros caprinos, os sinais foram mais uniformes e no início, consistiam em discreta perda de equilíbrio, quedas ocasionais, sonolência e reações posturais lentas. Essas alterações evoluíram gradativamente uma semana após o aparecimento dos primeiros sinais clínicos. Entre o 35ํ e 37ํ dia de experimento, os caprinos passaram a apresentar incoordenação motora com ataxia e hipermetria, posturas anormais, aumento da base de sustentação, perda de equilíbrio, dificuldade de se levantar, maior tempo em decúbito, andar cambaleante e quedas frequentes ao serem movimentados (Fig. 2). Emagrecimento progressivo, pêlos arrepiados, opacos e quebradiços também foram observados.

Nos Caprinos 7-9, não houve regressão dos sinais clínicos após a suspensão do fornecimento da planta.

À necropsia, não foram observadas alterações significativas, com exceção dos Caprinos 7-8, que apresentaram atrofia cerebelar. Nos caprinos do GII, ao exame histopatológico do SNC, as principais lesões foram encontradas no cerebelo, núcleos da base, bulbo na altura do óbex e telencéfalo. As lesões consistiam em vários graus de vacuolização do citoplasma de neurônios, sendo intensa em células de Purkinje (Fig. 3B) e discreta nos neurônios do córtex telencefálico. No SNC dos caprinos do GIII não foi observada vacuolização citoplasmática neuronal, as lesões eram discretas; foram observadas apenas no cerebelo, córtex frontal e parietal e consistiam em necrose de neurônios, evidenciada pela presença de corpos celulares de tamanho reduzido, com citoplasma eosinofílico e angular e núcleo picnótico. Em todos os caprinos experimentais, raros esferoides axonais foram observados na camada granular e substância branca do cerebelo e no bulbo na altura do óbex. Áreas focais de gliose também foram observadas nos núcleos da base e substância branca do cerebelo. Nas células acinares pancreáticas, foliculares da tireoide, hepatócitos e nas células epiteliais dos túbulos renais a vacuolização citoplasmática foi evidente apenas nos caprinos do GII.

\section{Morfometria cerebelar}

Os resultados das análises morfométricas estão dispostos no Quadro 1. Com relação ao número de neurônios de Purkinje foi observada uma redução estatisticamente significativa do número dessas células nos caprinos do Grupo I e III em relação aos caprinos do Grupo II e Grupo IV. Os caprinos do Grupo I e III apresentaram espessura da camada molecular mais delgada que os caprinos dos Grupos II e IV. Individualmente, observou-se que todos caprinos experimentais e naturalmente intoxicados apresentaram diferenças significativas em relação aos caprinos do Grupo IV (controles). Com relação à mensuração dos corpos celulares dos neurônios de Purkinje, os resultados demonstraram diferenças significativas entre todos os grupos,
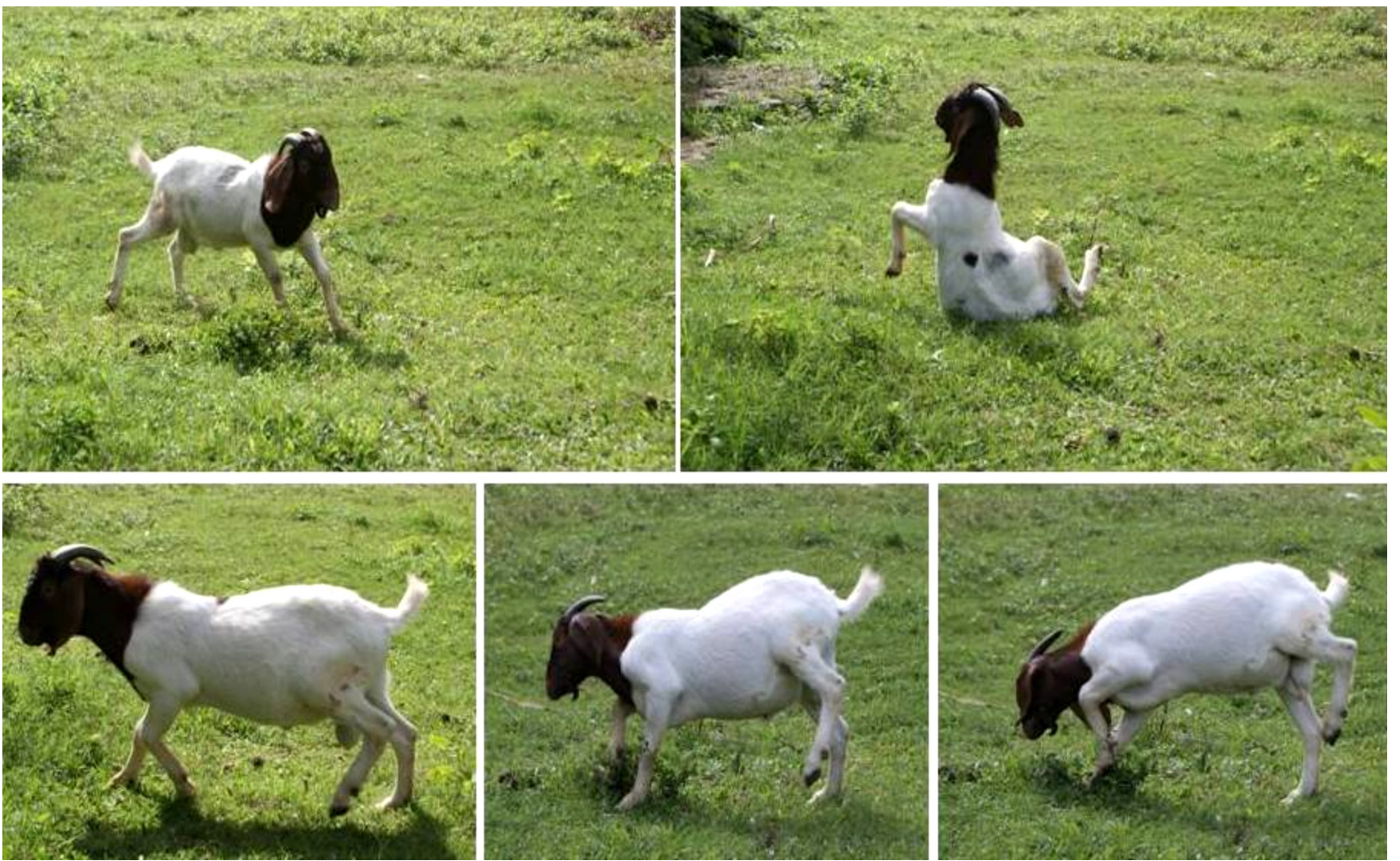

Fig.2. Doença de depósito lisossomal induzida pelo consumo de Ipomoea verbascoidea em caprinos. Caprino intoxicado experimentalmente, apresentando incoordenação motora, debilidade dos membros pélvicos, ataxia e quedas. 

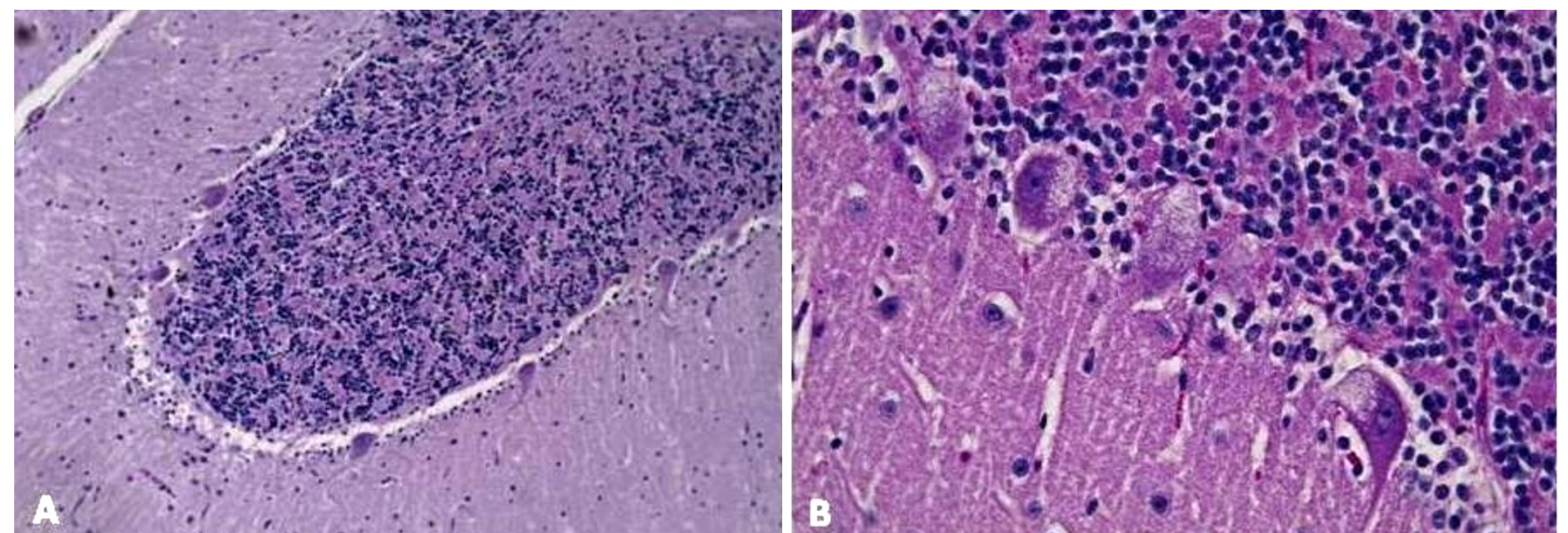

Fig.3. Doença de depósito lisossomal induzida pelo consumo de Ipomoea verbascoidea em caprinos. (A) Redução do tamanho e número de células na camada de neurônios de Purkinje em um caprino do Grupo III. HE, obj.10x. (B) Vacuolização citoplasmática dos neurônios de Purkinje e núcleos deslocados para as periferias das células em caprinos do grupo II. HE, obj.20x.

Quadro 1. Resultados da média, desvio padrão e coeficiente de variância do número de neurônios e área dos corpos celulares dos neurônios de Purkinje e espessura da camada molecular do cerebelo dos caprinos dos Grupos I- IV

\begin{tabular}{|c|c|c|c|c|c|c|c|c|c|c|c|c|}
\hline \multirow[t]{3}{*}{ Parâmetros } & \multicolumn{4}{|c|}{ № de neurônios de Purkinje } & \multirow{2}{*}{\multicolumn{4}{|c|}{$\begin{array}{l}\text { Área dos corpos celulares dos } \\
\text { neurônios de Purkinje (Sq } \mu \mathrm{m} \text { ) }\end{array}$}} & \multicolumn{4}{|c|}{ Espessura da camada molecular $(\mu \mathrm{m})$} \\
\hline & \multirow[t]{2}{*}{ Grupo I } & \multirow[t]{2}{*}{ Grupo II } & \multirow[t]{2}{*}{ Grupo III } & \multirow[t]{2}{*}{ Grupo IV } & & & & & \multirow[t]{2}{*}{ Grupo I } & \multirow[t]{2}{*}{ Grupo II } & \multirow[t]{2}{*}{ Grupo III } & \multirow[t]{2}{*}{ Grupo IV } \\
\hline & & & & & Grupo I & Grupo II & Grupo III & Grupo IV & & & & \\
\hline Média & $24^{\mathrm{a} *}$ & $31^{b}$ & $18,7^{\mathrm{a}}$ & $33,4^{b}$ & $329,1^{\mathrm{d}}$ & $844,8^{a}$ & $491,9^{c}$ & $547,4^{\mathrm{b}}$ & $239,5^{\mathrm{a}}$ & $349,3^{\mathrm{b}}$ & $293,4^{a}$ & $384,5^{\mathrm{b}}$ \\
\hline Desvio Padrão & 4 & 4,3 & 2,4 & 2,3 & 20,4 & 30,9 & 49,0 & 21,7 & 21,0 & 45,7 & 30,4 & 20,8 \\
\hline Coeficiente de Variância (\%) & 16,1 & 18,5 & 6 & 5,5 & 6,2 & 3,6 & 9,9 & 3,9 & 8,8 & 13 & 10,3 & 5,4 \\
\hline
\end{tabular}

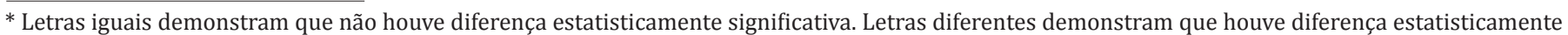
significativa em cada parâmetro entre os grupos estudados (Teste $t$ de student e Tukey $\mathrm{p}<0,05$ ).

sendo que os caprinos dos Grupos I e III apresentaram células com menor área. Os caprinos do Grupo II apresentaram corpos celulares maiores que os caprinos do Grupo IV. Na análise individual, foi evidenciado que as áreas dos corpos celulares de animais pertencentes ao mesmo grupo não apresentaram significância entre si. Porém, o Caprino 5 (Grupo II) apresentou dados diferentes em relação aos outros caprinos do mesmo grupo (Caprinos 4 e 6). Nesse caprinos a espessura da camada molecular, o número e área dos corpos celulares dos neurônios de Purkinje foram significativamente menores.

\section{DISCUSSÃO}

Os resultados apresentados nesse estudo sugerem que Ipomoea verbascoidea é uma planta tóxica de interesse pecuário em pelo menos quatro municípios do semiárido de Pernambuco. A planta foi encontrada somente no período chuvoso, cobrindo a vegetação herbácea e arbustiva ou crescendo em lajedos. A intoxicação ocorre no período chuvoso devido à intensa rebrotação de I. verbascoidea nessa época. Um fato que chama atenção na epidemiologia da intoxicação é que em 2012, não foram observados surtos da doença. Isto se deu, provavelmente, devido ao longo período de estiagem pelo qual passou a região semiária nordestina nesse ano.

No semiárido nordestino, outras plantas que contém swainsonina, tais como, I. carnea subsp. fistulosa, I. sericophylla, I. riedelii e Turbina cordata causam surtos de intoxicação principalmente em caprinos, com desenvolvimento de alterações neurológicas semelhantes e que estão associadas com armazenamento de oligossacarídeos (Barbosa et al. 2006, Riet-Correa et al. 2009, Mendonça et al. 2011, Oliveira et al. 2013). Os sinais clínicos induzidos por plantas que contém swainsonina são característicos principalmente de disfunção cerebelar; porém, lesões no bulbo, tais como as observadas nos caprinos deste estudo, também podem ser responsáveis pelas alterações proprioceptivas (Riet-Correa et al. 2002).

Em um estudo anterior, a doença de depósito lisossomal foi reproduzida em caprinos mediante o fornecimento de $I$. sericophylla dessecada em doses diárias de $2 \mathrm{~g} / \mathrm{kg}$ (1,5mg de swainsonina $/ \mathrm{kg}$ ) e de $\mathrm{I}$. riedelii dessecada em doses diárias de $2 \mathrm{~g} / \mathrm{kg}$ (0,6mg de swainsonina/kg). Nesse trabalho por um erro no cálculo da dose os autores informaram que as quantidades de planta ingeridas corresponderiam a 150mg e 60mg de swainsonina/kg (Barbosa et al. 2007). Quatro caprinos que continuaram ingerindo essas plantas por 39-89 dias após os primeiros sinais clínicos permaneceram com sequelas da doença, enquanto que dois caprinos que ingeriram as plantas por 15 e 20 dias após os primeiros sinais clínicos se recuperaram totalmente (Barbosa et al. 2007). Para conferir a recuperação das alterações posturais e de marcha provocadas por $I$. verbascoidea, a planta foi fornecida em doses contínuas de $0,8 \mathrm{mg} / \mathrm{kg}$ de swainsonina por 55 dias. Nenhum caprino se recuperou dos sinais clínicos. Esse resultado evidencia que o consumo da planta por 26-28 dias após a observação dos primeiros sinais clínicos já é suficiente para provocar lesões irreversíveis. 
As análises estatísticas relativas à mensuração da camada molecular do cerebelo, tanto da comparação individual entre os animais, como da comparação entre os grupos (I, II, III e IV), demonstraram probabilidade de erro de $1 \%$ $(\mathrm{p}<0,01)$ garantindo alta confiabilidade do teste utilizado. Na morfometria, houve variação considerável quanto ao tamanho dos corpos celulares e quanto ao número de neurônios de Purkinje entre os grupos estudados. No cerebelo dos caprinos dos Grupos I e III houve diminuição significativa do número de neurônios de Purkinje (Quadro 1). Resultados similares foram observados em caprinos intoxicados por I. carnea subsp. fistulosa onde a média de neurônios de Purkinje variou de 17 a 32 células por campo (Oliveira et al. 2011).

Nas doenças de depósito lisossomal, com a progressão das lesões, os neurônios apresentam um aspecto tumefeito e posteriormente sofrem necrose; os que sobrevivem à ação da swainsonina se tornam atróficos (Maxie \& Youssef 2007). Provavelmente, por isso, na análise morfométrica, os corpos celulares dos neurônios de Purkinje dos Caprinos do Grupo II, que estavam vacuolizados e tumefeitos tenham apresentado maior área. Nos caprinos dos Grupos I e III observou-se proporcionalidade entre a área do pericário dos neurônios e espessura da camada molecular do cerebelo. Os caprinos que apresentaram áreas de corpos celulares menores apresentaram camadas moleculares mais delgadas. A redução da espessura da camada molecular desses grupos pode também ser atribuída à necrose e desaparecimento de neurônios, principalmente os de Purkinje. Sugere-se que essas alterações sejam responsáveis pelo quadro clínico neurológico observado nos caprinos que deixam de ingerir a planta ou apresentam seqüelas da intoxicação.

Agradecimentos.- Trabalho financiado pelo Instituto Nacional de Ciência e Tecnologia Para o Controle das Intoxicações por Plantas (CNPq proc. 573534/2008-2).

\section{REFERÊNCIAS}

Assis T.S., Medeiros R.M.T., Riet-Correa F., Galiza G.J.N., Dantas A.F.M. \& Oliveira D.M. 2010. Intoxicações por plantas diagnosticadas em ruminantes e equinos e estimativa das perdas econômicas na Paraíba. Pesq. Vet. Bras. 30(1):13-20.

Balogh K.K.I.M., Dimande A.P., Lugt J.J., Molyneux R.J., Naudé T.W. \& Welman W.G.A. 1999. Lisosomal storage disease induced by Ipomoea carnea en goats in Mozambique. J. Vet. Diagn. Invest. 11(3):266-273.

Barbosa R.C., Riet-Correa F., Medeiros R.M.T., Lima E.F., Barros S.S., Gimeno E.J., Molyneux R.J. \& Gardner D.R. 2006. Intoxication by Ipomoea sericophylla and Ipomoea riedelii in goats in the state of Paraiba, Northeastern Brazil. Toxicon 47(4):371-379.

Barbosa R.C., Riet-Correa F., Lima E.F., Medeiros R.M.T., Guedes K.M.R, Gardner D.R., Molyneux R.J. \& Melo L.E.H. 2007. Experimental swainsonine poisoning in goats ingesting Ipomoea sericophylla and Ipomoea riedelii (Convolvulaceae). Pesq. Vet. Bras. 27(10):409-414.
Colodel E.M., Driemeier D., Loretti A.P., Gimeno E.J., Traverso S.D., Seitz A.L. \& Zlotowski P. 2002. Aspectos clínicos e patológicos da intoxicação por Sida carpinifolia (Malvaceae) em caprinos no Rio Grande do Sul. Pesq. Vet. Bras. 22(2):51-57.

Dantas A.F.M., Riet-Correa F., Gardner D.R., Medeiros R.M.T., Barros S.S., Anjos B.L. \& Lucena R.B. 2007. Swainsonine-induced lysosomal storage disease in goats caused by the ingestion of Turbina cordata in northeastern Brazil. Toxicon 49(1):11-16.

Gardner D.R, Molyneux R.J. \& Ralphs M.H. 2001. Analysis of swainsonine: Extraction methods, detection and measurement in populations of locoweeds (Oxytropis spp.). J. Agric. Food Chem. 49(10):4573-4580.

Guedes K.M.R., Riet-Correa F., Dantas A.F.M., Simões S.V.D., Miranda Neto E.G., Nobre V.M.T. \& Medeiros R.M.T. 2007. Doenças do sistema nervoso central em caprinos e ovinos no semi-árido. Pesq. Vet. Bras. 27(1): 29-38.

IBGE 2010. Fundação Instituto Brasileiro de Geografia e Estatística. <http:// http://www.ibge.gov.br/cidadesat/topwindow.htm?1> Acessado em 30 abr. 2013.

Maxie M.G. \& Youssef S. 2007. Nervous system, p.281-457. In: Maxie M.G. (Ed.), Jubb, Kennedy and Palmer's Pathology of Domestic Animals. Vol.1. $5^{\text {th }}$ ed. Saunders Elsevier, Philadelphia.

Mendonça F.S., Albuquerque R.F., Evêncio-Neto J., Freitas S.H., Dória R.G.S., Boabaid F.M., Driemeier D., Gardner D.R., Riet-Correa F. \& Colodel E.M. 2012.Alpha-mannosidosisingoats caused by theswainsonine-containing plant Ipomoea verbascoidea. J. Vet. Diagn. Invest. 24(1):90-95.

Mendonça F.S., Evêncio-Neto J., Estevão L.R.M., Albuquerque R.F. \& Colodel E.M. 2011. Spontaneous poisoning by Ipomoea sericophylla (Convolvulaceae) in goats at semi-arid region of Pernambuco, Brazil: a case report. Acta Vet. Brno 80(2):235-239.

Oliveira C.A., Barbosa J.D., Duarte M.D., Cerqueira V.D., Riet-Correa F., Tortelli F.P. \& Riet-Correa G. 2009. Intoxicação por Ipomoea carnea subsp. fistulosa (Convolvulaceae) em caprinos na Ilha do Marajó, Pará. Pesq. Vet. Bras. 29(7):583-588.

Oliveira C.A., Riet-Correa F., Dutra M.D., Cerqueira D.V., Araújo C.V. \& Riet-Correa G. 2011. Sinais clínicos, lesões e alterações produtivas e reprodutivas em caprinos intoxicados por Ipomoea carnea subsp. fistulosa (Convolvulaceae) que deixaram de ingerir a planta. Pesq. Vet. Bras. 31(11): 953-960.

Oliveira C.A., Riet-Correa G. \& Riet-Correa F. 2013. Intoxicação por plantas que contêm swainsonina no Brasil. Ciência Rural 43(4):653-661.

Pienaar J.G., Kellerman T.S., Basson P.A., Jenkins W.L. \& Vahrmeijer J. 1976. Maldronksiekte in cattle: a neuronopathy caused by Solanum kwebense N.E. Br. Onderstepoort J. Vet. Res. 43:67-74.

Riet-Correa F., Riet-Correa G. \& Schild A.L. 2002. Importância do exame clínico para o diagnóstico das enfermidades do sistema nervoso em ruminantes e equídeos. Pesq. Vet. Bras. 22:161-168.

Riet-Correa F., Pfister J., Schild A.L. \& Wierenga T. 2009. Poisonings by plants, mycotoxins and related substances in Brasilian livestock. Pallotti, Santa Maria. 246p.

Silva D.M., Riet-Correa F., Medeiros R.M.T. \& Oliveira O.F. 2006. Plantas tóxicas para ruminantes e equídeos no Seridó Ocidental e Oriental do Rio Grande do Norte. Pesq. Vet. Bras. 26(4):223-236.

Smith M.C. \& Sherman D.M. 2009. Nervous System, p.229-231. In: Smith M.C. \& Sherman D.M. (Eds), Goat Medicine. Williams and Wilkins, Iowa.

Tokarnia C.H., Brito M.F., Barbosa J.D., Peixoto P.V. \& Döbereiner J. 2012. Plantas Tóxicas do Brasil para Animais de Produção. 2a ed. Helianthus, Rio de Janeiro. 223p. 